\title{
BOUNDS ON THE SIGNED ROMAN $k$-DOMINATION NUMBER OF A DIGRAPH
}

\author{
Guoliang HaO \\ College of Science \\ East China University of Technology \\ Nanchang 330013, P.R. China \\ e-mail: guoliang-hao@163.com \\ XiaOdan Chen $^{1}$ \\ College of Mathematics and Information Science \\ Guangxi University \\ Nanning 530004, P. R. China \\ e-mail: x.d.chen@live.cn \\ AND \\ Lutz Volkmann \\ Lehrstuhl II für Mathematik \\ RWTH Aachen University \\ 52056 Aachen, Germany \\ e-mail: volkm@math2.rwth-aachen.de
}

\begin{abstract}
Let $k$ be a positive integer. A signed Roman $k$-dominating function (SRkDF) on a digraph $D$ is a function $f: V(D) \rightarrow\{-1,1,2\}$ satisfying the conditions that (i) $\sum_{x \in N^{-}[v]} f(x) \geq k$ for each $v \in V(D)$, where $N^{-}[v]$ is the closed in-neighborhood of $v$, and (ii) each vertex $u$ for which $f(u)=-1$ has an in-neighbor $v$ for which $f(v)=2$. The weight of an SRkDF $f$ is $\sum_{v \in V(D)} f(v)$. The signed Roman $k$-domination number $\gamma_{s R}^{k}(D)$ of a digraph $D$ is the minimum weight of an SRkDF on $D$. We determine the exact values of the signed Roman $k$-domination number of some special classes of digraphs and establish some bounds on the signed Roman $k$-domination number of general digraphs. In particular, for an oriented tree $T$ of order
\end{abstract}

\footnotetext{
${ }^{1}$ Corresponding author.
} 
$n$, we show that $\gamma_{s R}^{2}(T) \geq(n+3) / 2$, and we characterize the oriented trees achieving this lower bound.

Keywords: signed Roman $k$-dominating function, signed Roman $k$-domination number, digraph, oriented tree.

2010 Mathematics Subject Classification: 05C69, 05C20.

\section{REFERENCES}

[1] H.A. Ahangar, M.A. Henning, C. Löwenstein, Y. Zhao and V. Samodivkin, Signed Roman domination in graphs, J. Comb. Optim. 27 (2014) 241-255. doi:10.1007/s10878-012-9500-0

[2] Y. Caro and M.A. Henning, Directed domination in oriented graphs, Discrete Appl. Math. 160 (2012) 1053-1063. doi:10.1016/j.dam.2011.12.027

[3] G. Chartrand and L. Lesniak, Graphs and Digraphs, Fourth Ed. (Chapman and Hall, Boca Raton, FL, 2005).

[4] J. Cyman, Total outer-connected domination in trees, Discuss. Math. Graph Theory 3 (2010) 377-383. doi:/10.7151/dmgt.1500

[5] G. Hao and J. Qian, On the sum of out-domination number and in-domination number of digraphs, Ars Combin. 119 (2015) 331-337.

[6] F. Harary, R.Z. Norman and D. Cartwright, Structural Models (Wiley, New York, 1965).

[7] M.A. Henning and V. Naicker, Bounds on the disjunctive total domination number of a tree, Discuss. Math. Graph Theory 36 (2016) 153-171. doi:10.7151/dmgt.1854

[8] M.A. Henning and L. Volkmann, Signed Roman $k$-domination in trees, Discrete Appl. Math. 186 (2015) 98-105. doi:10.1016/j.dam.2015.01.019

[9] M.A. Henning and L. Volkmann, Signed Roman k-domination in graphs, Graphs Combin. 32 (2016) 175-190. doi:10.1007/s00373-015-1536-3

[10] S.M. Sheikholeslami and L. Volkmann, Signed Roman domination in digraphs, J. Comb. Optim. 30 (2015) 456-467. doi:10.1007/s10878-013-9648-2

[11] L. Volkmann, Signed Roman k-domination in digraphs, Graphs Combin. 32 (2016) 1217-1227. doi:10.1007/s00373-015-1641-3 
[12] B. Zelinka, Signed domination numbers of directed graphs, Czechoslovak Math. J. 55 (2005) 479-482.

doi:10.1007/s10587-005-0038-5

Received 23 November 2016

Revised 11 May 2017

Accepted 11 May 2017 